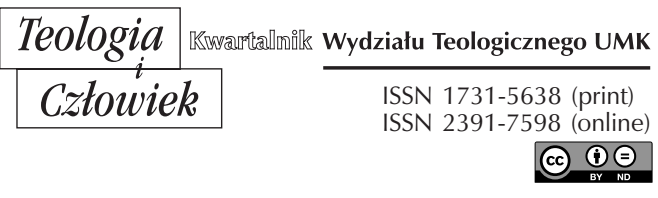

53(2021)1, ss. 71-94

KS. RYSZARD SELEJDAK

\title{
LA FORMAZIONE DEI CANDIDATI AL DIACONATO PERMANENTE SECONDO LE DISPOSIZIONI DELLA RATIO FUNDAMENTALIS INSTITUTIONIS DIACONORUM PERMANENTIUM
}

DOI: http://dx.doi.org/10.12775/TiCz.2021.004

Streszczenie. Formacja kandydatów do diakonatu stałego według wskazań Ratio fundamentalis institutionis diaconorum permanentium. Kościół za jedno z najważniejszych swoich zadań uważa zapewnienie kandydatom do diakonatu stałego właściwej formacji, odpowiadającej duchowi czasów. Szybkie bowiem zmieniające się warunki, w których odbywa się posługa diakońska, przemiany społeczno-kulturowe, rozwój nauk kościelnych i świeckich, rozwój życia chrześcijańskiego i diakońskiego na różnych etapach organizacji, wymagają od diakona solidnej formacji. Dokument zatytułowany Ratio fundamentalis institutionis diaconorum permanentium, opublikowany przez Kongregację Edukacji Katolickiej 22 lutego 1998 r., przedstawia formację kandydatów do diakonatu stałego jako proces wieloetapowy i wieloaspektowy. Program formacji zarówno kandydatów młodszych, jak i starszych ma trwać co najmniej trzy lata, nie licząc okresu propedeutycznego. Kandydaci młodsi do diakonatu powinni odbywać swoją formację w specjalnie założonych do tego celu krajowych lub dicezjalnych ośrodkach formacyjnych. W przypadkach nadzwyczajnych powinni oni brać udział w organizowanych kursach na odległość poprzez wykorzystanie różnych środków przekazu informacji. Jeśli chodzi o kandydatów starszych, zarówno żonatych, jak i celibatariuszy, Ratio fundamentalis nakazuje formację zgodnie z programem ustalonym przez Konferencję Biskupów. Modele formacji tej grupy osób muszą uwzględniać ich obowiązki rodzinne i zawodowe. 
Kandydaci przynależący do Instytutów życia konsekrowanego lub Stowarzyszeń życia apostolskiego są zobowiązani do odbycia formacji zgodnie z dyrektywami ewentualnego Ratio własnego Instytutu lub Stowarzyszenia. Mogą też skorzystać ze struktur formacyjnych diecezji, w której przebywają. Dokument Kongregacji zwraca uwagę, iż w formację diakonów winien być zaangażowany cały Kościół i odpowiedzialni za nią winni być biskupi lub wyżsi przełożeni zakonni, dyrektorzy formacji, opiekunowie kandydatów, kierownicy duchowni, proboszczowie, profesorzy, członkowie wspólnoty formacji diakonów stałych i członkowie wspólnoty pochodzenia kandydatów. Ponieważ formacja jest równocześnie autoformacją, szczególnym protagonistą, ponoszącym odpowiedzialność za własną formację, ma być sam kandydat do diakonatu, który świadomy swojej tożsamości i celu, ku któremu zmierza, musi stosować właściwe środki i metody do jego osiągnięcia.

Słowa kluczowe: sakrament Święceń; posługa święceń; diakonat stały; diakoni stali; teologia diakonatu; sakramentalność diakonatu; formacja diakonów stałych; diakonia liturgii; słowa i miłości; duchowość diakona; rozeznanie powołania; zdatność kandydatów; itinerarium i wymiary formacyjne; okres propedeutyczny.

\footnotetext{
Abstract. Formation of Candidates for the Permanent Diaconate According to the Instructions Contained in the Ratio Fundamentalis Institutionis Diaconorum Permanentium. The Church holds the view that one of her primary tasks is to ensure that candidates for the diaconate receive an adequate formation that corresponds to the spirit of the age. The rapidly evolving conditions in which the permanent diaconate finds itself, including cultural and social transformation, the advancement of ecclesial and secular sciences or the development of diaconal and Christian life at different stages require that a deacon should receive solid formation. The document Ratio fundamentalis institutionis diaconorum permanentium, published by the Congregation for Catholic Education on 22 February 1998, presents the formation of candidates for the permanent diaconate as a process that comprises various stages and dimensions. The programme of formation - excluding the preparatory period - is required to last at least three years for both young and mature candidates. The former must undertake their formation in national or diocesan centres of formation established for this very purpose; in exceptional cases, they may participate in distance learning courses by using various means of communication. In the case of the mature candidates, whether they be married or celibate, the Ratio fundamentalis envisions a programme of formation that would be decided by the Episcopal Conference. The models of formation for mature candidates must take into account their familial and professional commitments. Candidates who belong to Institutes of Consecrated Life or Societies of Apostolic Life are required to undergo formation in accord with the directives of the Ratio of their own Institute or Society, if applicable. They may also avail themselves of the programme that is available in their diocese of residence. The document of the Congregation underlines the fact that the entire ecclesial body ought to be involved in and responsible for the formation of deacons, namely: bishops, major superiors, directors of formation, tutors, spiritual directors, parish priests,
} 
professors, members of the community of formators for the permanent diaconate and members of the candidates' communities of origin. Given that formation is, at the same time, self-formation, the diaconal candidate himself is a necessary and irreplaceable agent in his own formation who - being aware of his own identity and the goal to which he aspires - must achieve that goal through appropriate means and methods.

Keywords: Sacrament of Holy Orders; ordained ministry; permanent diaconate; theology of the diaconate; sacramentality of the diaconate; formation of permanent deacons; diaconia of the liturgy, the word and charity; spirituality of the deacon; vocational discernment; suitability of candidates; formative itinerary and dimensions; propaedeutic period.

\section{INTRODUZIONE}

Il tempo della formazione come periodo preparatorio necessario prima di ricevere e esercitare un ministero ordinato in modo adeguato e rispondente alle circostanze odierne a favore del popolo di Dio, è tenuto in gran conto nella vita della Chiesa. Perciò il Magistero Ecclesiastico ha affrontato questo tema non soltanto riguardo ai futuri presbiteri, ma anche riguardo ai futuri diaconi permanenti ${ }^{1}$.

${ }^{1}$ Cf. P. Schiavone, Quale formazione per il diaconato permanente?, in: Il diaconato permanente, Napoli 1983, pp. 99-145; E. Petrolino, I diaconi annunziatori della Parola, ministri dell'altare e della carità, Cinisello Balsamo 1998, pp. 107-132; id., I diaconi per la chiesa del terzo millennio, in: La Chiesa diaconia universale di salvezza, a cura di G. Bellia, Reggio Emilia 2004, pp. 91-98; S. Zardoni, I diaconi nella Chiesa. Ricerca storica e teologica sul diaconato, Bologna 19912, pp. 172-177; J. S. Martins, Norme fondamentali per la formazione dei diaconi, Il diaconato in Italia 110 (1998), p. 47; R. Selejdak, Tożsamość, duchowość, formacja i posługa diakonów stałych, Częstochowa 2003, pp. 49-97; id., Diakonat stały w świetle dokumentów Soboru Watykańskiego II, pososborowego Urzędu Nauczycielskiego Kościoła i narodowych «Rationes institutionis diaconaorum permanentium», Warszawa 2010, pp. 185-274; M. Marczewski, Formacja diakonów stałych, Roczniki Teologiczne 47 (2000) 6, pp. 163-190; C. M. Martini, La diaconia come ministero della speranza, in: La Chiesa diaconia ministeriale della salvezza, a cura di G. Bellia, Reggio Emilia 2004, pp. 25-33; M. Presciutti-A. Farneti, La formazione, Orientamenti Pastorali 7 (2005), p. 173; R. Cabié, Le diaconat: un renouveau? Du diacre d'autrefois au diacre d'aujourd'hui, Bulletin de littérature ecclésiastique 107 (2006) 1, p. 112; J. Durán y Durán, Das Amt des Diakons im Dokument vov Aparecida 2007, Diaconia XP 43 (2008) 1-2, pp. 88-90; A. Montan, Il profilo del diaconato permanente nel 
Gli insegnamenti magisteriali contenuti nei documenti Gaudium et spes $^{2}$, Sollicitudo rei socialis ${ }^{3}$, Redemptoris missio ${ }^{4}$ e Pastores dabo vobis ${ }^{5}$ che presentano le caratteristiche del mondo contemporaneo che si possono riassumere attorno a questi punti focali:

- ci sono stati grandi cambiamenti scientifici, tecnici ed economici. L'energia nucleare è stata scoperta e utilizzata. Lautomazione sta progredendo costantemente, l'agricoltura e le aree rurali stanno cambiando, i mezzi di comunicazione si stanno sviluppando e i risultati nella conquista dello spazio sono eccellenti. Insieme a questi fenomeni, osserviamo profondi cambiamenti nella vita sociale. Cè un aumento del tenore di vita nei paesi ricchi e un calo simultaneo nei paesi del terzo mondo. Cè un aumento visibile del livello di istruzione e di varie forme di vita sociale;

- si risveglia un senso di uguaglianza sociale e politica in tutte le comunità e si ravvivano i contatti tra le nazioni, che prendono coscienza della loro reciproca dipendenza e del comune destino. si stanno formando Nel campo delleconomia, della scienza, dell'istruzione e della cultura si stanno formando comunità e organizzazioni internazionali e globali;

- risultati impressionanti in vari campi presenta l'annosa questione del significato della vita. Vi è una crescente necessità di regolamentare le questioni sociali su base etica, per superare la disarmonia, lo sfruttamento, la discriminazione e le minacce di guerra nucleare;

- un desiderio sempre più forte di giustizia e pace, la preoccu-

vigente Codice di Diritto Canonico, Seminarium 48 (2008) 4, pp. 765-766; I. Schinella, La dimensione intellettuale nella formazione diaconale, Seminarium 48 (2008) 4, p. 854.

${ }^{2}$ Cf. Sacrosanctum Concilium Oecumenicum Vaticanum II, Constitutio pastoralis de Ecclesia in mundo huius temporis Gaudium et spes, 5.12.1965, nn. 46-90, AAS 58 (1966), pp. 1066-1112.

${ }^{3}$ Cf. Giovanni Paolo II, Enciclica Solicitudo rei socialis, 30.12.1987, Libreria Editrice Vaticana 1987, nn. 11-26.

${ }^{4}$ Cf. id., Enciclica Redemptoris missio, 7.12.1990, Libreria Editrice Vaticana 1990, nn. 32-40.

${ }^{5}$ Cf. id., Esortazione apostolica post-sinodale circa la formazione dei sacerdoti nelle circostanze attuali Pastores dabo vobis, 25.03.1992, Libreria Editrice Vaticana 1992, nn. 6-9. 
pazione per la opera di creazione e rispetto per la natura, la ricerca della verità e protezione della dignità umana si fanno sempre più strada;

- alcune forme di religiosità delle sette continuano la loro diffusione;

- il razionalismo indebolisce la sensibilità alla Rivelazione e alla trascendenza divina. Vi è poi un'enfasi esagerata sulla soggettività della persona, la diffusione dell'ateismo politico ed esistenziale, il crollo della famiglia e lo stravolgimento della comprensione della sessualità umana;

- negli stessi ambienti ecclesiali preoccupano l'ignoranza religiosa di molti credenti, l'efficacia trascurabile della catechesi, un certo modo di condurre lo studio teologico, culturale e pastorale, la sfiducia nella gerarchia, la soggettivizzazione della fede, il fenomeno dell'appartenenza condizionale alla Chiesa;

- l'umanità parrebbe prepararsi a una nuova cultura sociale universale in cui tutti i popoli e le nazioni collaborino alla creazione del bene comune, alla costruzione della fratellanza, della civiltà e della cultura basate sui valori eterni.

Il 22 febbraio 1998, la Congregazione per l'Educazione Cattolica, tenendo presenti le suddette sfide del mondo, ha pubblicato la Ratio fundamentalis diaconorum permanentium ${ }^{6}$, documento di grande importanza per la formazione dei diaconi permanenti. Il Dicastero con esso intende non soltanto offrire alcuni principi di orientamento circa la formazione dei diaconi permanenti, ma anche dare alcune indicazioni che devono essere tenute in conto dalle Conferenze Episcopali nellelaborazione della Ratio nazionale. La Congregazione ha pensato di offrire agli Episcopati un strumento magisteriale, analogo alla Ratio fundamentalis institutionis sacerdotalis, per aiutarli ad adempire in modo adeguato le prescrizioni del can. 236, C.I.C., al fine di garantire alla Chiesa l'unità, la serietà e la completezza della formazione dei diaconi permanenti ${ }^{7}$.

${ }^{6}$ Cf. Congregatio de Institutione Catholica, Ratio fundamentalis institutionis diaconorum permanentium, 22.02.1998, AAS 90 (1998), pp. 843-879.

7 Cf. Congregatio de Institutione Catholica - Congregatio pro Clericis, De diaconatu permanenti, declaratio coniuncta, 22.02.1998, AAS 90 (1998), pp. 835-838. 
La Ratio fundamentalis diaconorum permanentium, è composta dall'Introduzione, da quattro capitoli riguardanti: i protagonisti della formazione dei diaconi permanenti, il profilo dei candidati al diaconato permanente, l'itinerario della formazione al diaconato permanente, le dimensioni della formazione dei diaconi permanenti e la conclusione.

\section{I DOCUMENTI PRECEDENTI ALLA RATIO SULLA FORMAZIONE DEI DIACONI PERMANENTI}

Circa la formazione dei diaconi permanenti, le prime indicazioni furono date dalla Lettera apostolica Sacrum diaconatus ordinem ${ }^{8}$. Esse sono state poi riprese e precisate nella Lettera circolare della Congrega-

${ }^{8}$ Cf. Paulus PP. VI, Litterae apostolicae motu date Sacrum diaconatus ordinem, 18.06.1967, AAS 59 (1967), pp. 697-704. La Lettera apostolica, al capitolo II, dedicato ai candidati giovani, prescrive: "6. I giovani candidati all'ufficio diaconale vengano accolti in un solo speciale istituto ove siano messi alla prova, educati a vivere una vita veramente evangelica e preparati a svolgere utilmente le proprie specifiche funzioni. 9. Il vero e proprio tirocinio diaconale si protragga almeno per la durata di tre anni; lordine degli studi, inoltre, sia regolato in modo che i candidati a grado a grado, progressivamente vengano disposti ad attendere con perizia ed utilità ai vari uffici diaconali. Nel suo complesso, poi, il ciclo degli studi potrà essere ordinato in modo tale che nel corso dell'ultimo anno venga data una specifica preparazione ai diversi uffici ai quali i diaconi, di preferenza, attenderanno. 10. A ciò si aggiungano le esercitazioni pratiche riguardanti l'insegnamento degli elementi della religione cristiana ai fanciulli e ad altri fedeli, la direzione e la divulgazione del canto sacro, la lettura dei libri divini della Scrittura nelle assemblee dei fedeli, la predicazione e l'esortazione al popolo, l'amministrazione dei sacramenti che competono ai diaconi, la visita agli ammalati e, in genere, ladempimento di quei servizi che ad essi possono essere commessi”. La medesima Lettera apostolica, al capitolo III, dedicato ai candidati di età matura, prescrive: "14. È auspicabile che anche tali diaconi siano provvisti di non mediocre dottrina, secondo quanto è stato detto ai nn. 8, 9, 10, o che almeno essi abbiano credito per quella preparazione intellettuale che, a giudizio della conferenza episcopale, sarà loro indispensabile per il compimento delle proprie specifiche funzioni. Siano perciò ammessi, per un certo tempo, in uno speciale istituto ove possano apprendere tutto ciò di cui avranno bisogno per attendere degnamente all'ufficio diaconale. 15. Che se ciò non possa farsi, l'aspirante venga affidato per l'educazione a qualche sacerdote di eminente virtù che si prenda cura di lui, lo istruisca e possa testimoniare, quindi, della di lui prudenza e maturità". 
zione per l'Educazione Cattolica Come è a conoscenza $a^{9}$, con cui si presentavano diversi tipi di formazione a seconda dei diversi tipi di diaconato (per celibi, sposati, destinati a luoghi di missione o a paesi ancora in via di sviluppo, chiamati ad esplicare la loro funzione in Nazioni di una certa civiltà e con una cultura abbastanza elevata. Per la formazione dottrinale si specificava che essa doveva essere al di sopra di quella di un semplice catechista e, in qualche modo, analoga a quella del sacerdote. Si elencavano poi, le materie che dovevano essere prese in considerazione per l'elaborazione del programma di studi ${ }^{10}$.

Paolo VI, nella successiva Lettera apostolica Ad pascendum precisò che "per quanto riguarda il corso degli studi teologici, che deve precedere l'ordinazione dei diaconi permanenti, è compito delle Conferenze Episcopali emanare, in base alle circostanze di luogo, le norme opportune, e sottoporle per l'approvazione alla Sacra Congregazione per l'Educazione Cattolica"11.

Il Codice di Diritto Canonico del 1983 integrò gli elementi essenziali di questa normativa nel can. $236^{12}$.

Dopo circa trent'anni dalle prime indicazioni, e con gli apporti delle esperienze successive, si è ritenuto opportuno elaborare la suddetta Ratio. Lo scopo di questo documento è quello di orientare ed armonizzare, nel rispetto delle legittime diversità, i programmi educativi tracciati dalle Conferenze Episcopali e dalle diocesi, che a volte risultano essere molto diversi tra di loro $^{13}$.

${ }^{9}$ Cf. Congregazione per l'Educazione Cattolica, Lettera circolare ai Rev.mi Rappresentanti Pontifici Come è a conoscenza sulla formazione dei candidati al diaconato permanente, Prot. N. 137/69, 16.07. 1969, EV 3 (1968-1970), pp. 834-837.

${ }^{10}$ Cf. ibidem, l.c., pp. 835-836.

11 Paulus PP.VI, Litterae apostolicae motu proprio datae nonnullae normae ad sacrum diaconatus ordinem spectantes statuuntur Ad pascendum, 15.08.1972, VII b, AAS 64 (1972), p. 540.

12 Cf. Ratio fundamentalis, n. 1: 1.c., p. 844.

13 Cf. ibidem, n. 2: 1.c., p. 844. 


\section{LA TEOLOGIA DEL DIACONATO}

La Ratio chiarisce che per una solida formazione dei diaconi permanenti è necessario avere una concezione teologica certa e solida del diaconato, tenendo conto della difficile comprensione della profonda realtà di questo ministero dovuta alla sua quasi totale scomparsa nella Chiesa d'Occidente per più di un millennio ${ }^{14}$. Il documento auspica che i riferimenti teologici possano essere ulteriormente sviluppati e approfonditi ${ }^{15}$. Il primo dato da sottolineare, a tale proposito, è quello ecclesiologico, per cui il diaconato va compreso come una precisa identità all'interno della Chiesa $^{16}$. È questo un riferimento necessario nella definizione dellessenza propria di ogni ministero ordinato, anche se non prioritario, in quanto la sua verità piena consiste nell'essere una partecipazione specifica ed una ripresentazione del ministero di Cristo ${ }^{17}$. Questa sottolineatura cristologica apre alla dimensione pneumatologica per cui il diaconato viene conferito mediante una speciale effusione dello Spirito, che realizza in chi la riceve una specifica conformazione a Cristo, Signore e servo di tutti ${ }^{18}$. La Ratio, ripresenta così quanto espresso nella Costituzione dogmatica sulla Chiesa Lumen gentium, n. 29, che a sua volta cita un testo delle Constitutiones Ecclesiae Aegyptiacae, secondo cui l'imposizione delle mani al diacono non è "ad sacerdotium sed ad ministerium", cioè, non per la celebrazione eucaristica, ma per il servizio. Questa indicazione, insieme al monito di San Policarpo, pure ripreso dalla Lumen gentium, n. $29^{19}$, delinea l'identità teologica specifica del diacono. Egli, partecipando nell'unico ministero ecclesiastico, è nella Chiesa segno sacramentale specifico di Cristo servo. Suo compito è di essere "interprete delle necessità e dei desideri delle comunità cristiane" e "animatore del servizio, ossia della diakonia"20, che

${ }^{14}$ Cf. ibidem, n. 3: 1.c., p. 844.

${ }^{15}$ Cf. ibidem.

${ }^{16}$ Cf. ibidem, n. 4: 1.c., p. 845.

17 Cf. ibidem.

18 Cf. ibidem, n. 5: 1.c., p. 845.

19 "Siano misericordiosi, attivi; camminino nella verità del Signore il quale si è fatto servo di tutti” (cf. Policarpo, Epistula ad Philippenses, 5, 2: Patres Apostolici, I, ed. F.X. Funk, Tubingae 1901, pp. 300-302).

${ }^{20}$ Ad pascendum, Introduzione: 1.c., pp. 534-538. 
è parte essenziale della missione della Chiesa ${ }^{21}$. Da un punto di vista sacramentale si ricorda che materia dell'ordinazione diaconale è l'imposizione delle mani del Vescovo, mentre la forma è costituita dalle parole della preghiera di ordinazione ${ }^{22}$. In quanto grado dellordine sacro, il diaconato imprime il carattere e comunica una grazia sacramentale specifica. Il carattere diaconale è il segno impresso indelebilmente nell'anima che configura chi è ordinato a Cristo, il quale si è fatto diacono, cioè servo di tutti ${ }^{23}$. Questo passaggio è di particolare rilevanza perché fino a quel momento i documenti magisteriali non parlavano esplicitamente di "un segno sacramentale di Cristo servo". Viene, pertanto, ribadita la sacramentalità del diaconato che in quanto grado del sacramento dellordine, imprime un carattere ed ha una propria specificità. Nell'esercizio delle loro funzioni, i diaconi, essendo partecipi ad un grado inferiore del ministero ecclesiastico, dipendono necessariamente dai Vescovi ${ }^{24}$. Inoltre, essi sono posti in una speciale relazione con i presbiteri, in comunione con i quali sono chiamati a servire il popolo di $\mathrm{Dio}^{25}$. Da un punto di vista canonico, con l'ordinazione, il diacono è incardinato nella Chiesa particolare o nella Prelatura personale al cui servizio è stato ammesso, oppure, in un Istituto religioso di vita consacrata o in una Società di vita apostolica ${ }^{26}$. Listituto dell'incardinazione non rappresenta un fatto accidentale, ma si caratterizza come legame stabile di servizio ad una concreta porzione di popolo di Dio. Esso implica l'appartenenza ecclesiale a livello giuridico, affettivo e spirituale e l'obbligo del servizio ministeriale ${ }^{27}$.

${ }^{21}$ Cf. Ratio fundamentalis, n. 5: 1.c., p. 846.

${ }^{22}$ Cf. ibidem, n. 6: 1.c., 846.

${ }^{23}$ Cf. ibidem, n. 7: 1.c., 846; cfr. anche Catechismo della Chiesa Cattolica, 11.10.1992, Città del Vaticano 1992. n. 1570.

${ }^{24}$ Cf. Ratio fundamentalis, n. 8: 1.c., p. 847.

${ }^{25}$ Cf. ibidem; cf. anche Sacrosanctum Concilium Oecumenicum Vaticanum II, Decretum de pastorali Episcoporum munere in Ecclesia Christus Dominus, 28.10.1965, n. 15, AAS 58 (1966), pp. 679-680.

${ }^{26}$ Cf. Ratio fundamentalis, n. 8: 1.c., p. 847; cfr. anche C.I.C., can. 266.

${ }^{27}$ Cf. Ratio fundamentalis, n. 8: 1.c., p. 847. 


\section{IL MINISTERO DEL DIACONO NEI DIVERSI CONTESTI PASTORALI E LA SUA SPIRITUALITÀ}

La specificità del ministero diaconale è spigata facendo riferimento alla triplice diaconia della liturgia, della Parola e della carità. La Ratio intende come ministero del diacono, nei diversi contesti pastorali ${ }^{28}$, le varie funzioni che egli può porre in essere secondo lo schema dei tria munera.

In riferimento al munus docendi, il diacono è chiamato a proclamare la Scrittura e istruire ed esortare il popolo ${ }^{29}$.

Il munus sanctificandi del diacono si esplica nella preghiera, nell'amministrazione solenne del battesimo, nella custodia e distribuzione dell'Eucaristia, nell'assistenza e benedizione del matrimonio, nella presidenza del rito del funerale e della sepoltura e nellamministrazione dei sacramentali ${ }^{30}$.

Infine, il munus regendi si esercita nella dedizione alle opere di bene e di assistenza e nell'animazione di comunità o settori della vita ecclesiale, specie per quanto riguarda la carità definita come il ministero più tipico del diacono ${ }^{31}$.

Quest'ultima espressione fortemente orientata all'azione di solidarietà e vicinanza non ha paralleli in altri testi ufficiali.

La Ratio, pertanto, sottolinea che sono diversi i modelli concreti dellesercizio del ministero diaconale, che dovranno essere suggeriti di volta in volta dalle diverse situazioni pastorali delle singole Chiese e saranno precisate dell'iter formativo proposto ${ }^{32}$.

Dall'identità teologica del diacono, scaturiscono con chiarezza i lineamenti della sua specifica spiritualità, che si presenta essenzialmente come quella del servizio ${ }^{33}$. Il modello per eccellenza è Cristo servo, vissuto

${ }^{28}$ Cf. ibidem, n. 9: 1.c., p. 847.

${ }^{29}$ Cf. ibidem; cfr. anche Sacrosanctum Concilium Oecumenicum Vaticanum II, Constitutio dogmatica de Ecclesia Lumen gentium, 21.11.1964, n. 29, AAS 57 (1965), p. 36.

${ }^{30}$ Cf. Ratio fundamentalis, n. 9: 1.c., pp. 847-848; cfr. anche Lumen gentium, n. 29, l.c., p. 36.

${ }^{31}$ Cf. Ratio fundamentalis, n. 9: 1.c., p. 848; cfr. anche Lumen gentium, n. 29: 1.c., p. 36 .

${ }^{32}$ Cf. Ratio fundamentalis, n. 10: 1.c., p. 848.

${ }^{33}$ Cf. ibidem, n. 11: 1.c., p. 848. 
totalmente al servizio di Dio, per il bene degli uomini. La spiritualità del servizio è di tutta la Chiesa, ma perché tutta la Chiesa possa meglio viverla, il Signore le dona un segno vivente e personale del suo stesso essere servo. Perciò, in modo specifico, essa è la spiritualità del diacono ${ }^{34}$. Egli, infatti, con la sacra ordinazione, è costituito nella Chiesa icona vivente di Cristo servo e nel farsi servitore generoso e fedele di Dio e degli uomini, specie dei più poveri e sofferenti consiste il suo impegno ascetico ${ }^{35}$.

\section{COMPITI DELLE CONFERENZE EPISCOPALI E DEI VESCOVI}

Particolare importanza rivestono le Conferenze Episcopali e i singoli Vescovi. Innanzitutto a ciascuna Conferenza Episcopale è data la competenza di attivare l'istituzione del diaconato come proprio e permanente grado della gerarchia, con l'assenso del Sommo Pontefice, in vista del bene dei fedeli ${ }^{36}$. Inoltre la Ratio, richiama il can. 236 del Codice di Diritto Canonico che attribuisce a tali organismi anche la competenza a specificare mediante disposizioni complementari la disciplina riguardante la recita della Liturgia delle Ore, l'età richiesta per l'ammissione e la formazione, ribadisce tali compiti ${ }^{37}$. Questo canone, infatti, stabilisce che siano le Conferenze Episcopali ad emanare, in base alle circostanze di luogo, le norme opportune perché i candidati al diaconato permanente, sia giovani sia di età più matura, sia celibi sia coniugati, siano formati a condurre una vita evangelica e siano preparati a compiere nel debito modo i doveri propri dellordine ${ }^{38}$.

Il documento specifica che pur restando il compito delle Conferenze Episcopali, la Congregazione per l'Educazione Cattolica ha preparato la Ratio, al fine di aiutarle a tracciare itinerari formativi che, pur attenti alle diverse situazioni particolari, siano tuttavia in sintonia con il cammino della Chiesa intera. Inoltre il testo intende offrire un punto di

${ }^{34}$ Cf. ibidem, n. 11: 1.c., pp. 848-849.

${ }^{35}$ Cf. ibidem.

${ }^{36}$ Cf. ibidem, n. 13: 1.c., p. 849; cfr. anche Sacrum diaconatus ordinem, n. 1: 1.c., p. 699.

${ }^{37}$ Cf. Ratio fundamentalis, n. 13: l.c., p. 849.

${ }^{38}$ Cf. ibidem. 
riferimento per meglio precisare i criteri del discernimento vocazionale e i vari aspetti della formazione ${ }^{39}$. Il documento stabilisce soltanto alcune linee fondamentali di carattere generale, che costituiscono la norma cui dovranno riferirsi le Conferenze Episcopali per l'elaborazione o l'eventuale perfezionamento delle loro rispettive rationes nazionali ${ }^{40}$, dove, senza mortificare la creatività e l'originalità delle Chiese particolari, vengono perciò indicati i principi e i criteri, sulla base dei quali la formazione dei diaconi permanenti può essere stabilita correttamente e in armonia con le altre comunità locali ${ }^{41}$.

Analogamente poi a quanto stabilisce l'insegnamento del Concilio Vaticano II per le rationes institutionis sacerdotalis ${ }^{42}$, il documento richiede alle Conferenze Episcopali che hanno "restaurato"43 il diaconato permanente di sottoporre le loro rispettive rationes institutionis diaconorum permanentium all'esame e all'approvazione della Santa Sede e specificamente alla Congregazione per il Clero dal 2013. Questo Dicastero eventualmente le approva, dapprima ad experimentum, e poi per un determinato numero di anni, in modo che siano garantite periodiche revisioni ${ }^{44}$.

Il documento specifica che la decisione sulla restaurazione del diaconato permanente in una Nazione non implica l'obbligo della sua attivazione in tutte le diocesi ${ }^{45}$. Spetta al Vescovo diocesano, dopo aver sentito il parere del Consiglio presbiterale e, se esiste, del Consiglio pastorale diocesano, procedere eventualmente al riguardo, tenendo conto delle necessità concrete e della situazione specifica della sua Chiesa particolare $^{46}$. Questa modalità di discernimento pone in campo, in modo sinodale, quegli organismi di comunione che aiutano il Vescovo nel governo della diocesi. Nel caso in cui il Vescovo scelga di restaurare il diaconato permanente, sarà sua cura promuovere unopportuna catechesi

${ }^{39}$ Cf. ibidem, n. 14: 1.c., pp. 849-850.

${ }^{40}$ Cf. ibidem, n. 14: l.c., p. 850.

${ }^{41}$ Cf. ibidem.

${ }^{42}$ Cf. Sacrosanctum Concilium Oecumenicum Vaticanum II, Decretum de institutione sacerdotali Optatam totius, 28.10.1965, n. 1, AAS 58 (1966), pp. 713-714.

${ }^{43}$ Cf. Ratio fundamentalis, nn. 15-16: 1.c., p. 850

${ }^{44}$ Cf. ibidem, n. 15: l.c., p. 850.

${ }^{45}$ Cf. ibidem, n. 16: l.c., p. 850.

${ }^{46}$ Cf. ibidem. 
al riguardo, sia tra i laici che tra i sacerdoti e i religiosi, in modo che il ministero diaconale sia compreso in tutta la sua profondità ${ }^{47}$. Tale disposizione è volta a far comprendere all'intera comunità le ragioni della decisione di restauro tale ministero e soprattutto perché nella diocesi si creino le disposizioni opportune per una accoglienza di esso e una sapiente valorizzazione. Inoltre, il Vescovo deve provvedere ad erigere le strutture necessarie allopera formativa e a nominare collaboratori idonei che lo coadiuvino come responsabili diretti della formazione, oppure, a seconda delle circostanze, si potrà avvalersi delle strutture formative di altre diocesi, o quelle regionali o nazionali ${ }^{48}$.

Il Vescovo, sulla base della ratio nazionale e dell'esperienza in atto, ha il compito di far redigere un apposito regolamento diocesano che deve essere periodicamente aggiornato ${ }^{49}$.

Ogni Istituto di vita consacrata o Società di vita apostolica che abbia ottenuto il diritto di ripristinare al suo interno il diaconato permanente deve poi garantire la formazione umana, spirituale, intellettuale e pastorale dei suoi candidati ${ }^{50}$. Tale Istituto o Società si dovrà impegnare a predisporre un proprio programma formativo che recepisca il carisma e la spiritualità propri dell'Istituto o della Società e, allo stesso tempo, sia in sintonia con la Ratio fundamentalis, specie per quanto riguarda la formazione intellettuale e pastorale ${ }^{51}$.

\section{I FORMATORI}

La Ratio poi presenta tutti quelli che partecipano nel processo formativo con la consapevolezza che nella cura della Chiesa per i suoi figli, il primo protagonista è lo Spirito di Cristo ${ }^{52}$.

\footnotetext{
${ }^{47}$ Cf. ibidem.

${ }^{48}$ Cf. ibidem, n. 16: 1.c., pp. 850-851.

${ }^{49}$ Cf. ibidem, n. 16: 1.c., p. 851.

${ }^{50}$ Cf. ibidem, n. 17: 1.c., p. 851.

${ }^{51}$ Cf. ibidem, n. 17: 1.c., pp. 851-852.

${ }^{52}$ Cf. ibidem, n. 18: 1.c., p. 852.
} 
Il primo segno e strumento dello Spirito di Cristo è il Vescovo proprio dei candidati (o il Superiore maggiore competente) ${ }^{53}$. È lui il responsabile ultimo del discernimento e della formazione ${ }^{54}$. Egli, pur potendo esercitare ordinariamente tale compito tramite i collaboratori che si è scelto, nondimeno si impegna, a conoscere personalmente quanti si preparano al diaconato ${ }^{55}$. Altre figure formative sono, poi: il direttore per la formazione, il tutore (dove il numero lo richiede), il direttore spirituale e il parroco o il ministro cui il candidato è affidato per il tirocinio diaconale ${ }^{56}$.

Il direttore per la formazione, nominato dal Vescovo, ha il compito di coordinare le varie persone impegnate nella formazione, di presiedere e animare tutta l'opera educativa nelle sue varie dimensioni, e di tenere i contatti con le famiglie degli aspiranti e dei candidati coniugati e con le loro comunità di provenienza. Inoltre, egli ha la responsabilità di presentare al Vescovo, dopo aver sentito il parere degli altri formatori, escluso il direttore spirituale, il giudizio di idoneità sugli aspiranti per la loro ammissione tra i candidati, e su quelli ammessi circa la loro promozione allordine del diaconato ${ }^{57}$. Nel procedere alla nomina del direttore, il Vescovo dovrà tener conto che sia un uomo di fede viva e di forte senso ecclesiale, abbia avuto un'ampia esperienza pastorale e abbia dato prova di saggezza, equilibrio e capacità di comunione, oltre che ad una solida competenza teologica e pedagogica. Potrà essere un presbitero o un diacono e, preferibilmente, non essere allo stesso tempo anche il responsabile per i diaconi già ordinati. Infatti, sarebbe auspicabile che questa responsabilità rimanesse distinta da quella per la formazione degli aspiranti e dei candidati ${ }^{58}$.

${ }^{53} \mathrm{Al}$ Vescovo diocesano sono equiparati in merito coloro ai quali sono affidate la prelatura territoriale, l'abbazia territoriale, il vicariato apostolico, la prefettura apostolica e l’amministrazione apostolica stabilmente eretta (cf. C.I.C., cann. 368; 381, \$2) nonché la prelatura personale (cf. ibidem, cann. 266, $\$ 1$; 295) e l'ordinariato militare (cf. Giovanni Paolo II, Costituzione apostolica Spirituali militum curae [21.04.1986], Libreria Editrice Vaticana [1986], art. I, $\$ 1$; art. II, $\$ 1$ ).

${ }^{54}$ Cf. Ratio fundamentalis, n. 19: 1.c., pp. 852-853.

${ }_{55}$ Cf. ibidem.

${ }^{56}$ Cf. ibidem, n. 20: 1.c., p. 853.

${ }^{57}$ Cf. ibidem, n. 21: 1.c., p. 853.

${ }^{58}$ Cf. ibidem. 
Il tutore, designato dal direttore per la formazione tra i diaconi o tra i presbiteri di provata esperienza e nominato dal Vescovo, è l'accompagnatore diretto di ogni aspirante e di ogni candidato. Egli ha l'incarico di seguire da vicino il cammino di ciascuno o di un gruppo, offrendo il suo sostegno e il suo consiglio per la soluzione degli eventuali difficoltà e per la personalizzazione dei vari momenti formativi ${ }^{59}$.

Il direttore spirituale è scelto liberamente da ogni aspirante o candidato e dovrà essere approvato dal Vescovo o dal Superiore maggiore ${ }^{60}$. Il suo compito è di accompagnare e sostenere la continua conversione dei chiamati, dare loro concreti suggerimenti per la maturazione di unautentica spiritualità diaconale e offrire stimoli efficaci per l'acquisizione delle virtù che vi sono connesse. Per tutto ciò, gli aspiranti e i candidati dovrebbero affidarsi per la direzione spirituale solo a sacerdoti di provata virtù, dotati di buona cultura teologica, di profonda esperienza spirituale, di spiccato senso pedagogico, di forte e squisita sensibilità ministeriale ${ }^{61}$.

Il direttore per la formazione d'accordo con l'équipe formativa e tenendo conto delle diverse situazioni dei candidati individua il parro$\mathrm{CO}^{62}$, chiamato ad offrire a colui che gli è stato affidato una viva comunione ministeriale e ad iniziarlo ed accompagnarlo nelle attività pastorali che riterrà più idonee. Inoltre, avrà cura di fare una periodica verifica del operato svolto con il candidato stesso e di comunicare l'andamento del tirocinio al direttore per la formazione ${ }^{63}$.

I professori concorrono in modo rilevante alla formazione dei futuri diaconi, alimentando la crescita di fede dei candidati e abilitando al compito di maestri del popolo di Dio. Per tale ragione, essi devono preoccuparsi non solo di far acquisire la necessaria competenza scientifica e una sufficiente capacità pedagogica, ma anche di testimoniare con la vita la Verità che insegnano ${ }^{64}$. Per poter armonizzare il loro specifico contributo con le altre dimensioni della formazione, è importante che essi siano disponibili, a seconda delle circostanze, a collaborare e confrontarsi

\footnotetext{
${ }^{59}$ Cf. ibidem, n. 22: 1.c., p. 854.

${ }^{60}$ Cf. ibidem, n. 23: l.c., p. 854.

${ }^{61}$ Cf. ibidem.

${ }^{62}$ Cf. ibidem, n. 24: l.c., pp. 854-855.

${ }^{63}$ Cf. ibidem.

${ }^{64}$ Cf. ibidem, n. 25: l.c., p. 855.
} 
con le altre persone impegnate nel processo formativo. In questo modo contribuiscono ad offrire ai candidati una formazione unitaria e li faciliteranno nella necessaria opera di sintesi ${ }^{65}$.

Gli aspiranti e i candidati al diaconato permanente costituiscono una specifica comunità ecclesiale che influisce profondamente sulla dinamica formativa. Gli incaricati della formazione devono pertanto preoccuparsi che tale comunità sia caratterizzata da profonda spiritualità, senso di appartenenza, spirito di servizio e slancio missionario, e abbia un programma ben preciso di incontri e di preghiera ${ }^{66}$.

Le comunità di provenienza degli aspiranti e dei candidati al diaconato devono accompagnare il cammino formativo con la preghiera, il rispetto, il buon esempio delle virtù domestiche e l'aiuto spirituale e materiale, soprattutto nei momenti difficili ${ }^{67}$.

Per quanto attiene gli aspiranti e i candidati sposati, particolare importanza è data alla famiglia e al coniuge ${ }^{68}$.

La comunità parrocchiale è chiamata ad accompagnare l'itinerario di ogni suo membro verso il diaconato con il sostegno della preghiera e un adeguato cammino di catechesi che, mentre sensibilizza i fedeli verso questo ministero, dà al candidato un valido aiuto per il suo discernimento vocazionale.

Anche quelle aggregazioni ecclesiali dalle quali provengono aspiranti e candidati al diaconato possono continuare ad essere per loro fonte di aiuto e di sostegno. Nello stesso tempo, devono mostrare rispetto per la chiamata ministeriale dei loro membri non ostacolando, bensì promovendo in loro la maturazione di una spiritualità e di una disponibilità autenticamente diaconali ${ }^{69}$.

\section{I CANDIDATI}

Particolare importanza viene data all'individuazione di coloro che potranno essere avviati al percorso formativo in vista del diacona-
${ }^{65}$ Cf. ibidem.
${ }^{66}$ Cf. ibidem, n. 26: 1.c., pp. 855-856.
${ }^{67}$ Cf. ibidem, n. 27: 1.c., p. 856.
${ }^{68}$ Cf. ibidem.
${ }^{69}$ Cf. ibidem. 
to. Tale problematica è affrontata dalla Ratio, facendo riferimento alle testimonianze provenienti dalla Chiesa antica ${ }^{70}$. I criteri per individuare coloro che sono idonei al ministero si riferiscono a quanto afferma il can. 1029, a proposito dei chierici in generale, ovvero che possono essere promossi agli Ordini soltanto quelli che hanno fede integra, sono mossi da retta intenzione, posseggono la scienza debita, godono buona stima, sono di integri costumi e di provate virtù e sono dotati di tutte quelle altre qualità fisiche e psichiche congruenti con l'Ordine che deve essere ricevuto $^{71}$. A queste qualità generali, la Ratio aggiunge alcune specifiche qualità umane e virtù evangeliche che sono richieste da questo specifico ministero: la maturità psichica, la capacità di dialogo e di comunicazione, il senso di responsabilità, la laboriosità, l'equilibrio e la prudenza ${ }^{72}$. Tra le virtù evangeliche hanno particolare rilevanza: la preghiera, la pietà eucaristica e mariana, un senso della Chiesa umile e spiccato, lamore alla Chiesa e alla sua missione, lo spirito di povertà, la capacità di obbedienza e di comunione fraterna, lo zelo apostolico, la disponibilità al servizio, la carità verso i fratelli ${ }^{73}$. Inoltre, i candidati al diaconato devono essere "vitalmente" ${ }^{74}$ inseriti in una comunità cristiana e aver già esercitato con lodevole impegno opere di apostolato ${ }^{75}$. Essi possono provenire da tutti gli ambiti sociali ed esercitare qualsiasi attività lavorativa o professionale purché essa non sia, secondo le norme della Chiesa e il prudente giudizio del Vescovo, sconveniente con lo stato diaconale ${ }^{76}$. Inoltre, tale attività deve essere "praticamente" conciliabile con gli impegni di formazione

${ }^{70}$ Cf. ibidem, n. 30: 1.c., p. 858. A tal riguardo la Ratio cita sia San Paolo (1 Tm 3, 8-10.12-13), sia la Didaché che la lettera ai Filippesi di San Policarpo.

${ }^{71}$ Cf. ibidem, n. 31: 1.c., pp. 858-859.

${ }^{72}$ Cf. ibidem, n. 32: l.c., p. 859.

${ }^{73}$ Cf. ibidem.

${ }^{74}$ Cf. ibidem, n. 33: l.c., p. 859. È da sottolineare l'uso del avverbio "vitaliter" riferito all'inserimento del candidato nella vita della Chiesa. Questo elemento si potrebbe definire di natura ecclesiologica poiché tale vocazione sorge nella Chiesa ed è finalizzata alla Chiesa. Non si tratta di una occasionale frequentazione o di un assiduo servizio, quanto di vitale inserimento.

${ }^{75}$ Cf. ibidem, n. 33: l.c., p. 859.

${ }^{76}$ Cf. ibidem, n. 34: l.c., p. 859. 
e l'effettivo esercizio del ministero ${ }^{77}$. Per quanto attiene il requisito dell'età, la Ratio, ricordando il can. $1031 \$ 2$, stabilisce che possono essere ordinati coloro che hanno compiuto i 25 anni per i candidati non sposati, e i 35 anni per coloro che sono sposati ${ }^{78}$. Infine i candidati devono essere liberi da irregolarità e impedimenti ${ }^{79}$.

La Ratio esplicita le caratteristiche richieste ai candidati a partire dal loro stato di vita, ovvero del fatto che siano sposati o no.

Per coloro che da giovani sono chiamati al diaconato sono obbligati ad osservare la legge del celibato ${ }^{80}$.

Nel caso di uomini coniugati, non bastano i 35 anni detà, ma occorre anche una vita matrimoniale che duri da molto tempo, una vita familiare veramente cristiana e l'onesta reputazione ${ }^{81}$. Inoltre, è condizione necessaria allammissione tra i candidati, il consenso della moglie e la presenza in lei di naturali qualità che non vadano ad impedire o a disonorare il ministero del marito ${ }^{82}$.

Una ulteriore categoria di candidati è data da coloro che sono stati individuati in età più matura, anche essi sono tenuti al celibato ecclesiastico in virtù della tradizionale disciplina ecclesiastica ${ }^{83}$.

Lo stesso principio vale per i diaconi rimasti vedovi: essi sono chiamati a dare prova di solidità umana e spirituale nella loro condizione di vita. Inoltre, condizione perché i candidati vedovi possano essere accolti è che essi abbiano già provveduto o dimostrino di essere in grado di provvedere adeguatamente alla cura umana e cristiana dei loro figli ${ }^{84}$.

77 Cf. ibidem. Quest'ultima sottolineatura costituisce un interessante criterio di discernimento poiché oltre alle doti umane e propensioni ecclesiali, deve sussistere una reale possibilità in termini di tempo da dedicare e di funzioni da assolvere che siano conciliabili con lo stato familiare, lavorativo e sociale del candidato. Per cui un candidato, per esempio, che, pur presentando delle caratteristiche umane positive, non avesse molto tempo a disposizione a motivo del suo lavoro, non sarebbe idoneo al ministero.

78 Cf. ibidem, n. 35: 1.c., pp. 859-860.

${ }^{79}$ Cf. ibidem, n. 35: 1.c., p. 860. La Ratio fundamentalis specifica in nota tali irregolarità e impedimenti facendo riferimento ai cann. 1040-1042.

${ }^{80}$ Cf. ibidem, n. 36: 1.c., p. 860.

${ }^{81}$ Cf. ibidem, n. 37: 1.c., pp. 860-861.

${ }^{82}$ Cf. ibidem, n. 37: 1.c., p. 861.

${ }^{83}$ Cf. ibidem, n. 38: 1.c., p. 861.

${ }^{84}$ Cf. ibidem. 
I diaconi permanenti appartenenti a Istituti di vita consacrata o a Società di vita apostolica sono chiamati ad arricchire il loro ministero con il particolare carisma ricevuto armonizzando la vocazione religiosa o consacrata con quella ministeriale e ad offrire il loro originale contributo alla missione della Chiesa. Essi per quanto attiene la loro azione pastorale sono sottoposti all'Ordinario del $\operatorname{luog}{ }^{85}$.

\section{L'ITINERARIO E LE DIMENSIONI FORMATIVE}

La Ratio dedica ampio spazio alla fase formativa e alle attenzioni che devono essere oggetto dell'azione educativa. L'itinerario formativo è scandito da alcuni periodi: quello del discernimento previo; poi il propedeutico; e, in fine, il tempo della formazione specifica al ministero.

Il periodo di formazione propriamente detta è preceduto da una fase che si può chiamare di "discernimento previo". Tale fase riguarda la presentazione del candidato. Infatti, la decisione di intraprendere l'itinerario della formazione diaconale può avvenire o per iniziativa dell'aspirante stesso o per una esplicita proposta della comunità cui l'aspirante appartiene. In ogni caso, tale decisione deve essere accolta e condivisa dalla comunità ${ }^{86}$. A nome della comunità, il parroco (o il Superiore, nei casi di religiosi) deve presentare al Vescovo (o al Superiore maggiore competente) l'aspirante al diaconato illustrando le motivazioni che la sostengono, con un curriculum vitae $e^{87}$. La prima fase vede come protagonisti le comunità ecclesiali con i loro responsabili (parroci o Superiori) e i candidati individuati dalla comunità stessa. In seconda battuta il Vescovo (o il Superiore maggiore competente), dopo aver consultato il direttore per la formazione e l'équipe educativa, ha il compito di decidere circa l'ammissione al secondo periodo ovvero quello "propedeutico"88.

Il periodo propedeutico attraverso una più approfondita conoscenza della teologia, della spiritualità e del ministero diaconale serve

\footnotetext{
${ }^{85}$ Cf. ibidem, n. 39: 1.c., pp. 861-862.

${ }^{86}$ Cf. ibidem, n. 40: l.c., p. 862.

87 Cf. ibidem.

${ }^{88}$ Cf. ibidem.
} 
per operare un più attento discernimento sulla vocazione dei candidati ${ }^{89}$. Tale periodo ha come responsabile il direttore per la formazione che, a seconda dei casi, potrà affidare gli aspiranti ad uno o più tutori $i^{90}$. Si auspica la formazione di una comunità, con un proprio ritmo di incontri e di preghiera. In questo periodo sono valorizzate la direzione spirituale e il tirocinio pastorale ${ }^{91}$. In questo periodo la Ratio, non prevede lezioni scolastiche, ma incontri di preghiera, istruzioni, momenti di riflessione e di confronto orientati a favorire l'obiettività del discernimento vocazionale coinvolgendo, per quanto possibile, anche le spose degli aspiranti ${ }^{92}$. Alla fine del periodo propedeutico, il direttore per la formazione, dopo aver consultato l'équipe educativa e tenendo conto di tutti gli elementi in suo possesso, presenta al Vescovo (o al Superiore maggiore competente) un attestato che tracci il profilo della personalità degli aspiranti e, su richiesta, anche un giudizio di idoneità ${ }^{93}$. Il Vescovo (o il Superiore maggiore competente) qualora abbia raggiunto la certezza morale dell'idoneità dei candidati può procedere al rito liturgico di ammissione tra i candidati all'ordine del diaconato ${ }^{94}$. L'ascrizione tra i candidati al diaconato, specifica la Ratio, non costituisce alcun diritto a ricevere necessariamente l'ordinazione diaconale. Essa è un primo riconoscimento ufficiale dei segni positivi della vocazione al diaconato, che deve essere confermato nei successivi anni della formazione ${ }^{95}$.

Il periodo della formazione specifica deve durare almeno tre anni, oltre al periodo propedeutico, per tutti i candidati ${ }^{96}$. Per i candidati giovani si prescrive che ricevano la loro formazione dimorando per tre anni in una casa specifica ${ }^{97}$, mentre per i candidati di età più matura, sia celibi che coniugati, si prescrive che essi ricevano la loro formazione mediante un progetto formativo della durata di tre anni, determinato dalla Conferenza

\footnotetext{
${ }^{89}$ Cf. ibidem, n. 41: l.c., p. 862.

${ }^{90}$ Cf. ibidem, n. 42: 1.c., p. 863.

${ }^{91}$ Cf. ibidem.

${ }^{92}$ Cf. ibidem, n. 43: l.c., p. 863.

93 Cf. ibidem, n. 44: l.c., p. 863.

${ }^{94}$ Cf. ibidem, n. 45: l.c., p. 864.

95 Cf. ibidem, n. 48: 1.c., p. 864.

96 Cf. ibidem, n. 49: 1.c., pp. 864-865.

${ }^{97}$ Cf. ibidem, n. 50: 1.c., p. 865.
} 
Episcopale ${ }^{98}$. Esso deve essere attivato, dove le circostanze lo permettono, nel contesto di una viva partecipazione alla comunità dei candidati, con un proprio calendario di incontri di preghiera, di formazione e fraternità ${ }^{99}$. Per questi candidati sono possibili diversi modelli di organizzazione della formazione che tengano conto degli impegni lavorativi e familiari.

Per i candidati appartenenti agli Istituti di vita consacrata o alle Società di vita apostolica, la formazione venga fatta secondo le direttive dell'eventuale Ratio del proprio Istituto o della propria Società, oppure utilizzando le modalità della diocesi in cui i candidati si trovano ${ }^{100}$.

Il programma della formazione teologicamente ben fondato, deve avere una specifica finalizzazione pastorale, essere adattato alle necessità e ai programmi ecclesiali locali ${ }^{101}$ e deve tener conto delle diverse dimensioni formative: umana, spirituale, teologica e pastorale ${ }^{102}$. Litinerario deve coinvolgere, nelle forme che si riterranno opportune, le mogli e i figli dei candidati coniugati e così pure le loro comunità di appartenenza ${ }^{103}$. In questo periodo viene conferito il ministero del lettorato e dell'accolitato ${ }^{104}$. Alla fine dell'itinerario formativo, il candidato che, d'accordo con il direttore per la formazione, ritenga di avere i requisiti necessari per essere ordinato, può indirizzare al Vescovo proprio o al Superiore maggiore competente una dichiarazione, redatta e firmata di suo pugno, nella quale attesta che intende ricevere il sacro ordine spontaneamente e liberamente e si dedicherà per sempre al ministero ecclesiastico ${ }^{105}$. Il Vescovo o il Superiore maggiore, dopo aver raccolto l'attestato che il direttore per la formazione ha redatto ${ }^{106}$ ed eventualmente operando il discernimento opportuno può procedere con il rito di ordinazione ${ }^{107}$.

\footnotetext{
98 Cf. ibidem, n. 51: 1.c., p. 865.

${ }^{99}$ Cf. ibidem.

100 Cf. ibidem, n. 52: 1.c., pp. 865-866.

101 Cf. ibidem, n. 55: l.c., p. 866.

102 Cf. ibidem, nn. 66-88: 1.c., pp. 869-878.

103 Cf. ibidem, n. 56: 1.c., p. 866.

104 Cf. ibidem, nn. 57-59: l.c., pp. 866-867.

105 Cf. ibidem, n. 60: 1.c., pp. 867-868.

106 Cf. ibidem, n. 62: 1.c., p. 868.

107 Cf. ibidem, n. 64: 1.c., p. 869.
} 


\section{CONCLUSIONE}

Il documento della Congregazione per l'Educazione Cattolica intitolato Ratio fundamentalis institutionis diaconorum permanentium presenta la formazione dei candidati al diaconato permanente come un processo che abbraccia varie tappe e dimensioni. Poiché la formazione è allo stesso tempo autoformazione, protagonista necessario e insostituibile, responsabile della propria formazione, è il candidato stesso al diaconato. Il programma di formazione sia per i candidati giovani che per quelli di età più matura deve durare almeno tre anni, escluso il periodo introduttivo. I giovani candidati al diaconato devono seguire la loro formazione nei centri formativi nazionali o diocesani appositamente istituiti a questo scopo. Per i candidati adulti, sia sposati che celibi, il suddetto documento prescrive la formazione secondo un programma stabilito dalla Conferenza Episcopale. I candidati appartenenti a Istituti di vita consacrata o a Società di vita apostolica sono tenuti a seguire la formazione secondo le direttive delleventuale Ratio del proprio Istituto o della propria Società. Possono anche utilizzare le modalità di formazione della diocesi in cui si trovano. La Ratio fundamentalis institutionis diaconorum permanentium sottolinea che l'intera Chiesa dovrebbe essere coinvolta nella formazione dei diaconi e responsabile di essa.

\section{BIBLIOGRAFIA}

Cabié R., Le diaconat: un renouveau? Du diacre d'autrefois au diacre d'aujourd'hui, Bulletin de littérature ecclésiastique 107 (2006) 1, pp. 105-121.

Catechismo della Chiesa Cattolica, 11.10.1992, Città del Vaticano 1992.

Codice di Diritto Canonico, 3.02.1983, Città del Vaticano 1983.

Congregazione per l'Educazione Cattolica, Lettera circolare ai Rev.mi Rappresentanti Pontifici Come è a conoscenza sulla formazione dei candidati al diaconato permanente, Prot. N. 137/69, 16.07.1969: EV 3 (1968-1970), pp. 834-837.

Congregatio de Institutione Catholica, Ratio fundamentalis institutionis diaconorum permanentium, 22.02.1998, AAS 90 (1998), pp. 843-879.

Congregatio de Institutione Catholica - Congregatio pro Clericis, De diaconatu permanenti, declaratio coniuncta, 22.02.1998, AAS 90 (1998), pp. 835-842.

Durán y Durán J., Das Amt des Diakons im Dokument vov Aparecida 2007, Diaconia XP 43 (2008) 1-2, pp. 83-94. 
Giovanni Paolo II, Costituzione apostolica Spirituali militum curae, 21.04.1986, Libreria Editrice Vaticana 1986.

Giovanni Paolo II, Enciclica Solicitudo rei socialis, 30.12.1987, Libreria Editrice Vaticana 1987.

Giovanni Paolo II, Enciclica Redemptoris missio, 7.12.1990, Libreria Editrice Vaticana 1990.

Giovanni Paolo II, Esortazione apostolica post-sinodale circa la formazione dei sacerdoti nelle circostanze attuali Pastores dabo vobis, 25.03.1992, Libreria Editrice Vaticana 1992.

Durán y Durán J., Das Amt des Diakons im Dokument vov Aparecida 2007, Diaconia XP 43 (2008) 1-2, pp. 83-94.

Marczewski M., Formacja diakonów stałych, Roczniki Teologiczne 47 (2000) 6, pp. $163-190$.

Martini C. M., La diaconia come ministero della speranza, in: La Chiesa diaconia ministeriale della salvezza, a cura di G. Bellia, Reggio Emilia 2004, pp. 25-33.

Martins J. S., Norme fondamentali per la formazione dei diaconi, Il diaconato in Italia 110 (1998), pp. 46-53.

Montan A., Il profilo del diaconato permanente nel vigente Codice di Diritto Canonico, Seminarium 48 (2008) 4, pp. 751-785.

Paulus PP. VI, Litterae apostolicae motu date Sacrum diaconatus ordinem, 18.06.1967, AAS 59 (1967), pp. 697-704.

Paulus PP.VI, Litterae apostolicae motu proprio datae nonnullae normae ad sacrum diaconatus ordinem spectantes statuuntur Ad pascendum, 15.08.1972, AAS 64 (1972), pp. 534-540.

Petrolino E., I diaconi annunziatori della Parola, ministri dellaltare e della carità, Cinisello Balsamo 1998.

Petrolino E., I diaconi per la chiesa del terzo millennio, in: La Chiesa diaconia universale di salvezza, a cura di G. Bellia, Reggio Emilia 2004, pp. 91-98.

Policarpo, Epistula ad Philippenses, Patres Apostolici, I, ed. F.X. Funk, Tubingae 1901.

Presciutti M. - Farneti A., La formazione, Orientamenti Pastorali 7 (2005), pp. 173-179.

Sacrosanctum Concilium Oecumenicum Vaticanum II, Constitutio dogmatica de Ecclesia Lumen gentium, 21.11.1964, AAS 57 (1965), pp. 5-67.

Sacrosanctum Concilium Oecumenicum Vaticanum II, Decretum de institutione sacerdotali Optatam totius, 28.10.1965, AAS 58 (1966), pp. 713-727.

Sacrosanctum Concilium Oecumenicum Vaticanum II, Decretum de pastorali Episcoporum munere in Ecclesia Christus Dominus, 28.10.1965, AAS 58 (1966), pp. 673-701.

Sacrosanctum Concilium Oecumenicum Vaticanum II, Constitutio pastoralis de Ecclesia in mundo huius temporis Gaudium et spes, 5.12.1965, AAS 58 (1966), pp. 1025-1115.

Schinella I., La dimensione intellettuale nella formazione diaconale, Seminarium 48 (2008) 4, pp. 849-898. 
Schiavone P., Quale formazione per il diaconato permanente?, in: Il diaconato permanente, Napoli 1983, pp. 99-145.

Selejdak R., Tożsamość, duchowość, formacja i posługa diakonów stałych, Częstochowa 2003.

Selejdak R., Diakonat stały w świetle dokumentów Soboru Watykańskiego II, posoborowego Urzędu Nauczycielskiego Kościoła i narodowych «Rationes institutionis diaconaorum permanentium», Warszawa 2010.

Zardoni S., I diaconi nella Chiesa. Ricerca storica e teologica sul diaconato, Bologna $1991^{2}$. 